\title{
Synthesizing Asymmetric Side Lobe Pattern with Steered Nulling in Nonuniformly Excited Linear Arrays by Controlling Edge Elements
}

\author{
Jafar Ramadhan Mohammed and Khalil H. Sayidmarie \\ College of Electronics Engineering, Ninevah University, Mosul 41001, Iraq \\ Correspondence should be addressed to Khalil H. Sayidmarie; kh.sayidmarie@gmail.com
}

Received 12 January 2017; Revised 2 April 2017; Accepted 16 April 2017; Published 24 May 2017

Academic Editor: Jaume Anguera

Copyright (C) 2017 Jafar Ramadhan Mohammed and Khalil H. Sayidmarie. This is an open access article distributed under the Creative Commons Attribution License, which permits unrestricted use, distribution, and reproduction in any medium, provided the original work is properly cited.

\begin{abstract}
In radar antennas, asymmetric side lobes are useful, where undesired signals such as noise and ground clutter should be minimized. Also, for practical implementation, the feeding network of such antennas should be efficiently designed. In this paper, a simple analytical method for synthesizing asymmetric side lobe pattern with a wide-angle steered null in the nonuniformly excited linear arrays is presented. In this method, the difference in the side lobe levels on both sides of the main beam is achieved by varying just the phase excitations of the two-edge elements. The major novelty of this paper lies in the fact that the required asymmetric side lobe pattern can be achieved by changing a single phase shifter resulting in a simple feeding network.
\end{abstract}

\section{Introduction}

The performance of the radar and communication systems is highly affected by the level of the interfering signals and ground clutter that need to be rejected. In general, the rejection is achieved by synthesizing an array with low side lobe level or pointing the nulls towards the directions of interfering signals or clutter returns. Thus, the topic of side lobe reduction or cancellation has attracted much interest in the past and is still of great interest especially with the currently crowded spectrum.

From the computational complexity of the feed network point of view, the methods of side lobe canceling can be grouped into two categories. The first is fully controlled arrays [1-7] that require full control of weights of all the array elements, while the second is the partially controlled arrays that require few elements' weight to be controllable [8]. In both groups, it is much easier to control the phase distribution of the element excitations than to control the amplitude distribution $[9,10]$. To synthesize an array pattern with specific constraints on the side lobe structure, various optimization algorithms have been proposed in the literature.
These are particle swarm optimization [11, 12], genetic algorithm [13, 14], and simulated annealing [15] or the convex programming $[16,17]$. However, most of these optimization algorithms support the concept of fully controlled arrays, where the amplitude and phase excitations of all or most of the array elements are under control. This leads to a complex and expensive feeding network.

A simple method for null steering by controlling the amplitude and phase excitations of just the two-edge elements has previously been described in [18] for uniformly excited linear arrays. In this paper, the method is further extended to obtain a very general strategy that is applicable to uniformly and nonuniformly excited arrays. Moreover, a new and simple analytical model for producing asymmetric pattern with a large difference between the side lobe levels on both sides of the main beam is presented. The amplitude of the two-edge elements is adjusted initially; then by properly changing the phase excitations of the two-edge elements of the array antenna the asymmetric side lobe pattern can be implemented, and the side lobe nulling can be steered to the left or right side of the main beam. 


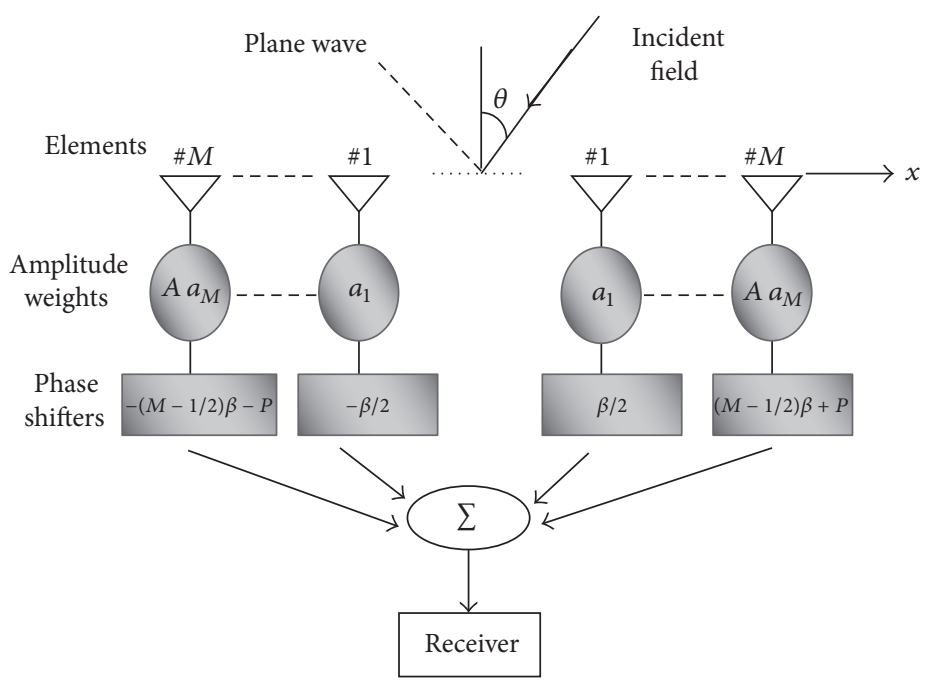

Figure 1: Block diagram of the proposed technique.

\section{Problem Formulation}

Consider a nonuniformly excited linear array antenna of $N=$ $2 M$ isotropic radiating elements placed symmetrically along $x$-axis and uniformly separated by a distance of $d=\lambda / 2$. The resulting symmetric normalized array factor is [19]

$$
A F(\theta)=\sum_{n=1}^{M} a_{n} \cos \left[\left(n-\frac{1}{2}\right) k d \sin (\theta)+\left(n-\frac{1}{2}\right) \beta\right]
$$

where $k$ is the wavenumber, $a_{n}$ are the excitation coefficients of the array elements, $\beta$ is the progressive phase difference between consecutive array elements, and $\theta$ is the observation angle from ( $z$-axis) the normal to array axis and varies from $-90^{\circ}$ to $90^{\circ}$. To find analytically the element excitations $a_{n}$ that are required for a given side lobe pattern, there are several array synthesizing methods such as Dolph-Chebyshev and Taylor [19]. However, these conventional synthesizing methods can only produce a symmetric beam pattern with specific side lobe level.

The idea is to generate asymmetric side lobe level with a capability of null steering in an array, whose radiation pattern is defined by (1), by suitably modifying the amplitude and phase excitations of just the two-edge elements while maintaining the element excitations of the inner elements unchanged. The two-edge elements have a radiation pattern that is aligned and out of phase with the side lobe pattern of the inner elements array. Then the total radiation pattern in the far field is the summation of the pattern due to twoedge elements and the pattern due to inner elements $(N-2$ elements). More details of this analytical model can be seen in the next section.

\section{Asymmetric Side Lobe Pattern}

The array factor of the nonuniformly excited array which consists of $N=2 M$ elements was given in (1), whereas the radiation pattern due to the two-edge elements alone can be written as

$$
\begin{aligned}
& A F_{2}(\theta) \\
& \quad=2 a_{M} \cos \left[\left(M-\frac{1}{2}\right) k d \sin (\theta)+\left(M-\frac{1}{2}\right) \beta\right] .
\end{aligned}
$$

As each edge element is located at a distance equal to $(M-$ $1 / 2) d$ from the center of the array, the pattern of the two-edge elements has grating lobes rather than side lobes.

Now we consider the case where the excitations of the two-edge elements in the nonuniformly excited array (shown in Figure 1) are modified such that the amplitude is scaled by a factor $A$, and a phase shift $P$ is added between the two-edge elements. Then, the far field pattern of the total $N$-elements array (including both subarrays, the $N-2$ inner elements array and two-edge elements array) after modification of the two-side elements will be

$$
\begin{aligned}
A F_{T} & (\theta) \\
= & \underbrace{\sum_{n=1}^{M-1} a_{n} \cos \left[\left(n-\frac{1}{2}\right)[k d \sin (\theta)+\beta]\right]}_{N-2 \text { elements array without edge elements }} \\
& +\underbrace{2 a_{M} A \cos \left[\left(M-\frac{1}{2}\right)[k d \sin (\theta)+\beta]-P\right]}_{\text {two-edge elements alone }} .
\end{aligned}
$$

It is wanted to set the values of $A$ and $P$ so that the side lobe structure of the two-edge elements is exactly identical to that of the $N-2$ inner elements array pattern. To get asymmetric side lobe level, the side lobe structures of these two subarrays should be in phase in one side of the main beam and out of phase in the other side of the main beam.

The amplitude excitation of the two-edge elements is found at the design stage depending on the design side lobe 

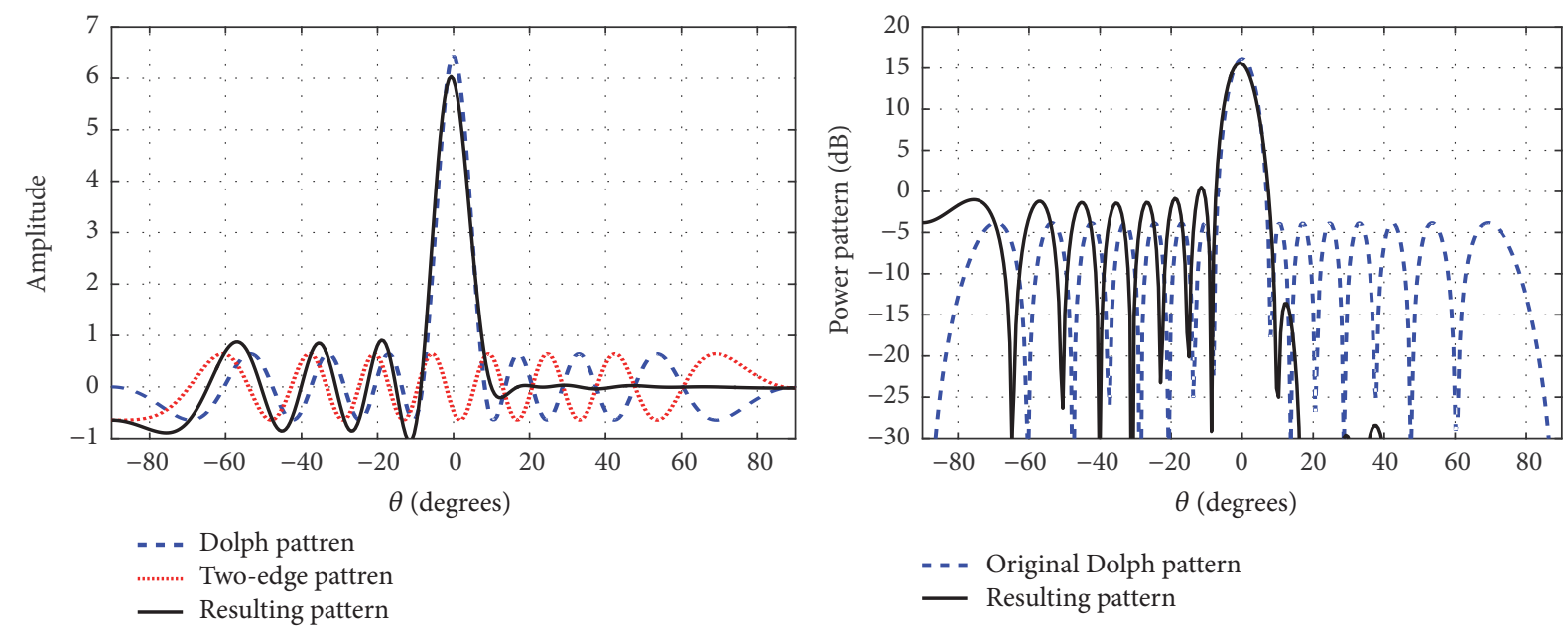

FIGURE 2: Radiation patterns of the original Dolph-Chebyshev array and the proposed arrays for $N=16, d=\lambda / 2$, and $P=9.1576^{\circ}$.

level; then the required change in the amplitude excitations of the two-edge elements can be easily found from the following:

$$
A=\frac{\mathrm{SLL}_{\mathrm{Req}}}{2 a_{M}},
$$

where $\mathrm{SLL}_{\text {Req }}$ represents the required side lobe level in the synthesized stage of the nonuniformly excited array pattern and $a_{M}$ is the original amplitude excitation of the edge elements in a known nonuniformly excited array.

Next, the required value of $P$ can be easily found from (3) by first specifying the prescribed direction of the jamming signal or clutter return, that is, $\theta=\theta_{\text {jammer }}$, and then putting $A F_{T}\left(\theta_{\text {jammer }}\right)=0$. Thus (3) becomes a function of a single unknown parameter. This analytical property makes the proposed method converge much faster than any other global optimization methods such as particle swarm optimization and genetic algorithm.

Choosing $P$ by this way not only creates a null at jammer direction but also cancels or reduces most of the side lobes in one side of the main beam by virtue of side lobe structure matching in both subarrays that are represented by (3). Finally, knowing $P$, the modified phase excitations of the twoedge elements which are located at positions $-(M-1 / 2) d$ and $(M-1 / 2) d$ in the nonuniformly excited linear array are $-(M-1 / 2) \beta-P$ and $(M-1 / 2) \beta P$.

\section{Simulation Results}

To validate the effectiveness and find the convergence speed of the proposed method, a number of examples have been performed on a $2.4 \mathrm{GHz}$ Laptop equipped with $2 \mathrm{~GB}$ of RAM. In the following, the Dolph-Chebyshev and Taylor distributions as examples for nonuniformly excited arrays are considered. In all cases, a linear array with $d=\lambda / 2$ and various numbers of array elements $(N)$ was assumed.

The first example starts with Dolph-Chebyshev excited array with designed SLL $=-20 \mathrm{~dB}$ and $N=16$ elements. Also, suppose that the jammer direction is at $\theta=65^{\circ}$. The far field radiation patterns of the original Dolph-Chebyshev array, the two-edge elements, and the resulting total pattern according to (3) are shown in Figure 2. Here, the value of $P$ was found to be $P=9.1576^{\circ}\left(9.16^{\circ}\right)$. From this figure, it can be seen that the radiation pattern of the two-edge elements is matched well with that of the original Dolph-Chebyshev array across a wide angular range centered around $\theta=65^{\circ}$ and extending to many side lobes on right side of the main beam. Thus, most of the side lobes in the right-hand side in the resulting pattern have been reduced down by more than $25 \mathrm{~dB}$ with respect to the original Dolph-Chebyshev pattern. Moreover, the required null direction centered at $\theta_{\text {jammer }}=65^{\circ}$ has been successfully fulfilled. On the other hand, the left-hand side of the resulting pattern has a peak side lobe level equal to $-15 \mathrm{~dB}$ (i.e., an increase in its side lobe level by $5 \mathrm{~dB}$ with respect to the original Dolph-Chebyshev array pattern). In this example, we obtain a gap of $30 \mathrm{~dB}$ between the side lobes at both sides of the main beam. An improvement of more than $10 \mathrm{~dB}$ over the results reported in $[9,10]$ has been noticed. The main beam widths in the original Dolph array and the proposed array patterns are $6.7520^{\circ}$ and $7.26^{\circ}$, respectively, while the taper efficiency has changed from $96.09 \%$ to $86.86 \%$. Figure 3 shows the amplitude and phase distributions of the original Dolph-Chebyshev array and the proposed array which are responsible for the synthesized patterns of Figure 2. It is clear that only the phase excitation of the two-edge elements has been modified. As mentioned earlier, the amplitude excitation of the two-edge elements is adjusted initially at the design stage of the Dolph-Chebyshev array.

In the second example, the number of array elements is chosen to be $N=30$ and we also started from DolphChebyshev excited array with designed SLL $=-20 \mathrm{~dB}$ as the previous example. Here, the prescribed null direction is centered at $\theta_{\text {jammer }}=-30^{\circ}$ and the value of $P$ is computed from (3) to be $18.7121^{\circ}$. Figures 4 and 5 show the resulting patterns and the corresponding amplitude and phase excitations, respectively. Again, from these results, it can be seen 

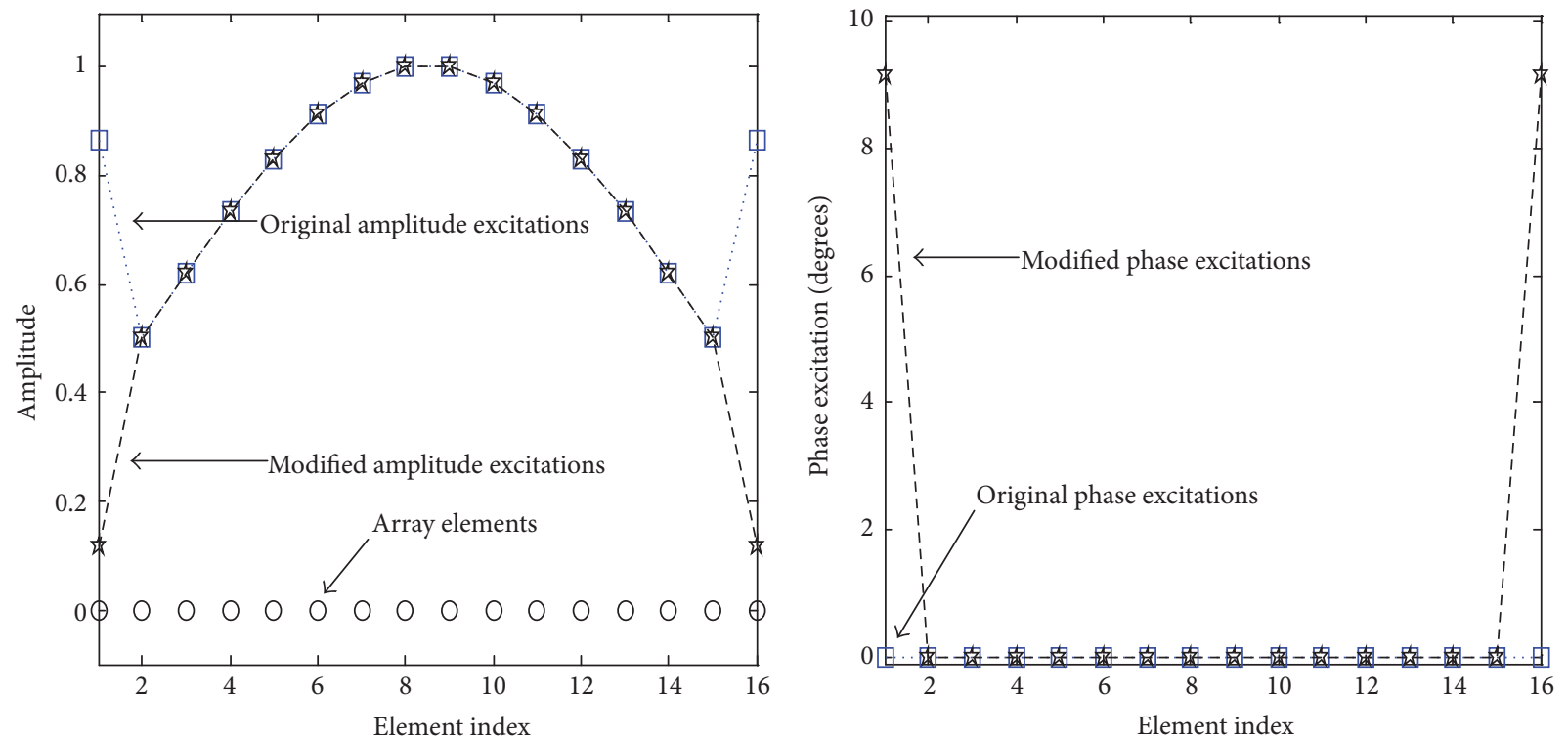

FIgUre 3: Amplitude and phase distributions of the original Dolph-Chebyshev array and the proposed arrays for $N=16$ and $d=\lambda / 2$.
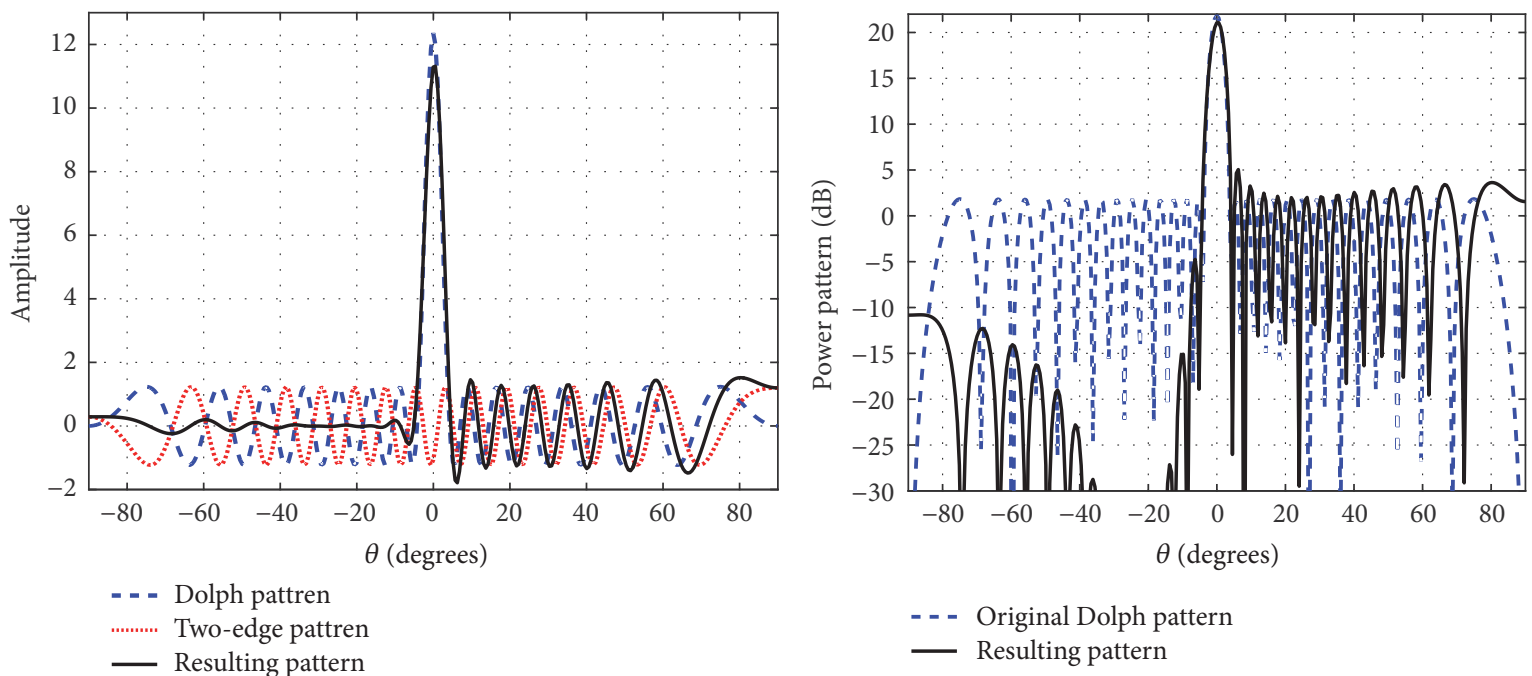

FIgURE 4: Radiation patterns of the original Dolph-Chebyshev array and the proposed arrays for $N=30, d=\lambda / 2$, and $P=18.7121^{\circ}$.

that the gap in side lobe between both sides of the main beam is $20 \mathrm{~dB}$ and the proposed array has achieved the desired objectives by placing a wide null at exactly the prescribed jammer direction. For further quantitative evaluation, the directivity, half-power beam width, taper efficiency, runtime, and peak side lobe level are computed and compared for both original Dolph array and the proposed array as shown in Table 1. Generally, the directivity and taper efficiency have been slightly decreased, while the HPBW is slightly increased.

Furthermore, two new performance evaluation parameters are introduced. The first one is the area under the side lobe regions (AUSL), while the second one is the area under the main beam (AUMB). The percentage difference in the area under main beams of the proposed array and the original Dolph-Chebyshev array patterns can be written as

$$
\Delta \mathrm{AUMB}=\frac{\left|\mathrm{AUMB}_{\text {Proposed }}-\mathrm{AUMB}_{\text {Dolph }}\right|}{\mathrm{AUMB}_{\text {Dolph }}} .
$$

On the other hand, the percentage difference in the area under side lobes for the proposed array and the original Dolph-Chebyshev array patterns can be written as

$$
\Delta \mathrm{AUSL}=\frac{\left|\mathrm{AUSL}_{\text {Proposed }}-\mathrm{AUSL}_{\text {Dolph }}\right|}{\mathrm{AUSL}_{\text {Dolph }}} .
$$



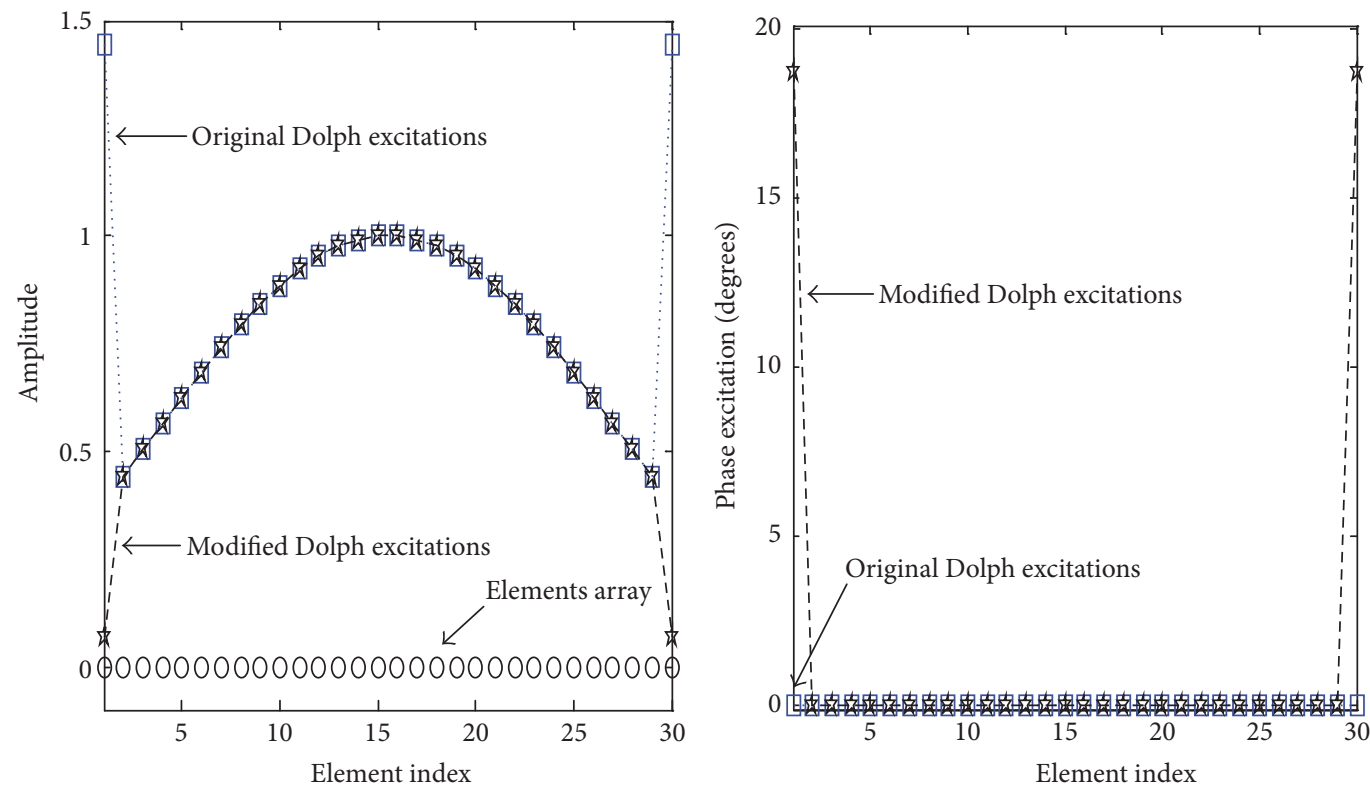

FIgUre 5: Amplitude and phase distributions of the original Dolph-Chebyshev array and the proposed arrays for $N=30$ and $d=\lambda / 2$.

TABLE 1: Patterns features for different number of array elements $N$ and $d=\lambda / 2$.

\begin{tabular}{|c|c|c|c|c|c|c|}
\hline \multirow[t]{2}{*}{ Features } & \multicolumn{3}{|c|}{ Original Dolph array pattern } & \multicolumn{3}{|c|}{$\begin{array}{l}\text { Proposed array pattern } \\
\text { (change with respect to original Dolph pattern) }\end{array}$} \\
\hline & $N=10$ & $N=16$ & $N=30$ & $N=10$ & $N=16$ & $N=30$ \\
\hline Directivity $[\mathrm{dB}]$ & 9.8441 & 11.8794 & 14.4222 & $\begin{array}{c}9.5102 \\
(-3.34 \%)\end{array}$ & $\begin{array}{l}11.5210 \\
(-3.02 \%)\end{array}$ & $\begin{array}{l}14.1047 \\
(-2.2 \%)\end{array}$ \\
\hline HPBW [deg] & 11.1520 & 6.7520 & 3.5200 & $\begin{array}{l}11.8200 \\
(5.98 \%)\end{array}$ & $\begin{array}{c}7.26 \\
(7.5 \%)\end{array}$ & $\begin{array}{l}3.8700 \\
(9.9 \%)\end{array}$ \\
\hline Taper efficiency & 0.9622 & 0.9609 & 0.9207 & $\begin{array}{c}0.8404 \\
(-12.6 \%)\end{array}$ & $\begin{array}{c}0.8686 \\
(-9.6 \%)\end{array}$ & $\begin{array}{c}0.8955 \\
(-2.7 \%)\end{array}$ \\
\hline Runtime [second] & 1.75756 & 1.83548 & 1.87657 & $\begin{array}{c}1.75756 \\
(0.0 \%)\end{array}$ & $\begin{array}{c}1.83548 \\
(0.0 \%)\end{array}$ & $\begin{array}{r}1.87657 \\
(0.0 \%)\end{array}$ \\
\hline Peak SLL $[\mathrm{dB}]$ & -20 & -20 & -20 & $\begin{array}{c}-15.1 \\
(4.9 \mathrm{~dB})\end{array}$ & $\begin{array}{c}-15.7 \\
(4.3 \mathrm{~dB})\end{array}$ & $\begin{array}{c}-16.7 \\
(3.3 \mathrm{~dB})\end{array}$ \\
\hline AUSL & 0.0442 & 0.0531 & 0.0683 & 0.0343 & 0.0290 & 0.0238 \\
\hline$\triangle$ AUSL $\%$ & - & - & - & $(-22.4 \%)$ & $(-45.38 \%)$ & $(-65.15 \%)$ \\
\hline AUMB & 0.262 & 0.1796 & 0.0917 & 0.2890 & 0.1935 & 0.0981 \\
\hline$\triangle \mathrm{AUMB} \%$ & - & - & - & $(10.3 \%)$ & $(7.74 \%)$ & $(6.97 \%)$ \\
\hline
\end{tabular}

These areas are computed and compared for various scenarios as shown in Table 1 . As expected, the value of $\mathrm{AUMB}_{\text {Proposed }}$ is slightly larger than that of $\mathrm{AUMB}_{\text {Dolph }}$, while $\mathrm{AUSL}_{\text {Proposed }}$ is much smaller than that of AUSL ${ }_{\text {Dolph. This fully confirms }}$ the effectiveness of the proposed array. More importantly, the value of $\triangle \mathrm{AUSL}$ is increasing with larger number of array elements $(N)$, while the value of $\triangle \mathrm{AUMB}$ is decreasing.

In the next example, we start from a Taylor excited array with designed SLL $=-20 \mathrm{~dB}$ and $N=16$ elements. The resulting patterns are shown in Figure 6 which clearly shows that the required null position centered at $\theta_{\text {jammer }}=20^{\circ}$ has been achieved and a gap of $20 \mathrm{~dB}$ in the side lobe between both sides of the main beam has been obtained. Here, the value of $P$ was found to be $P=6.75^{\circ}$.

Finally, for a real examination, we used a 16 -element linear array of half-wavelength dipoles over a simulated finite ground plane based on wire grid model as shown in Figure 7(a) and obtained active element patterns using $4 \mathrm{NEC} 2$. $4 \mathrm{NEC} 2$ is an efficient tool for modeling these thin dipoles in free space and exploring the array performance, including the effects of mutual coupling. In this structure, we assumed a vertical array of 16 horizontal half-wavelength 


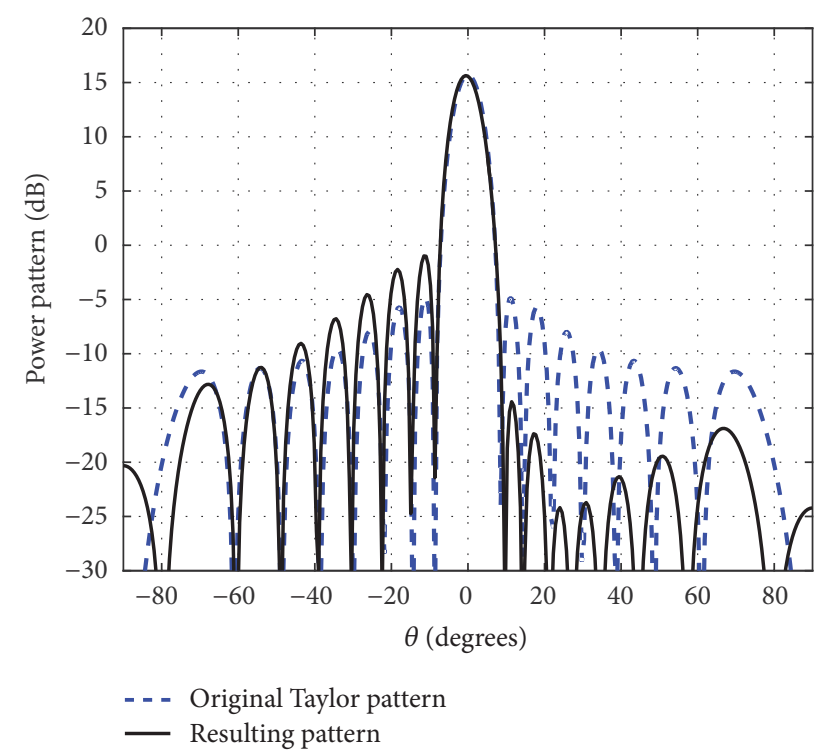

FIgURE 6: Radiation patterns of the original Taylor pattern and the proposed arrays for $N=16, d=\lambda / 2$, and $P=6.75^{\circ}$.

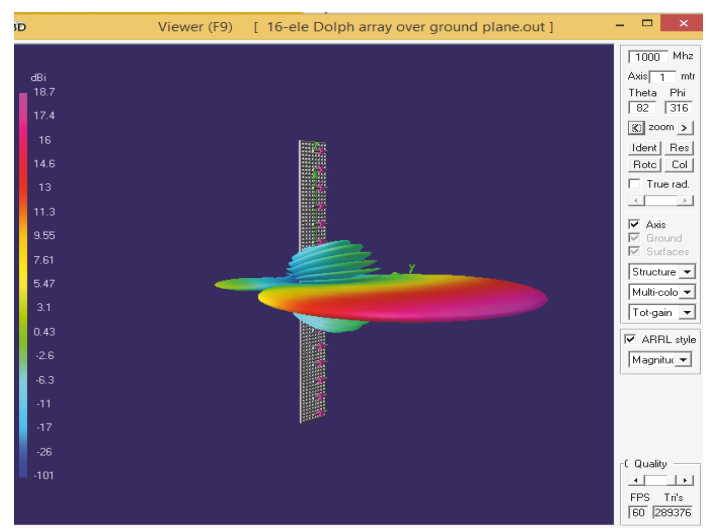

(a)

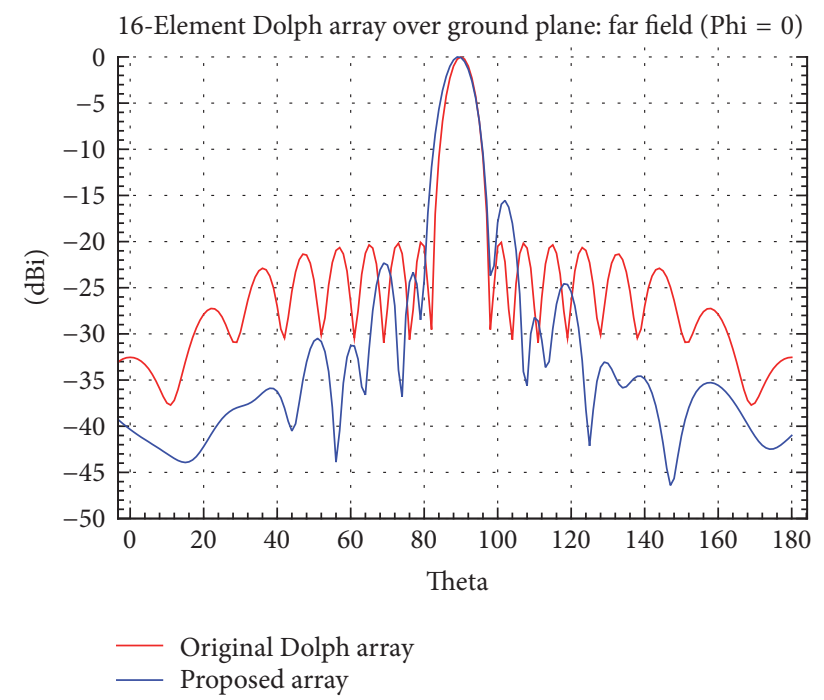

(b)

FIGURE 7: (a) Configuration of an array with 16 dipoles over ground plane. (b) Radiation patterns of the original Dolph pattern and the proposed arrays. Center frequency $=1 \mathrm{GHz}$. dipoles (for the design frequency of $1 \mathrm{GHz}$; then the length of each dipole in free space is equal to $150 \mathrm{~mm}$ ). Such arrangement of horizontal dipoles will have significant broadside coupling between the elements.

In the 4NEC2 model of the Dolph excited array, each dipole was divided into 21 segments and a voltage source assigned to the center segment (segment 11) of each dipole. This allowed the specific amplitude and phase excitations to be applied to the individual elements. In this model, the Dolph-Chebyshev excited array with designed SLL = $-20 \mathrm{~dB}$ and $N=16$ dipoles is considered. Figure $7(\mathrm{~b})$ shows the original Dolph pattern and the resulting pattern with asymmetric side lobe level. These patterns are, of course, different from those in Figure 2 due to the mutual coupling effect and the active element pattern.

\section{Conclusions}

The problem of producing wide tunable nulls along with asymmetric side lobe pattern in nonuniformly excited linear arrays using simple analytical model has been addressed. The wide null and asymmetric side lobe pattern are accomplished by reusing edge elements of the array whose radiation pattern approximates that of the side lobe pattern of the main array over a wide angular range. The proposed array was applied to a Dolph-Chebyshev distribution as well as Taylor distribution and achieved differences in the side lobe level on both sides of the main beam as great as $20 \mathrm{~dB}$. Almost same beam width and directivity were noticed, as compared with original Dolph-Chebyshev arrays. The other advantage of the proposed analytical method over the global optimization methods is its ability to offer faster solution and simpler implementation. The basic principle of the proposed method can be extended to other nonuniformly excited linear or planar arrays.

\section{Conflicts of Interest}

The authors declare that they have no conflicts of interest.

\section{References}

[1] R. A. Monzingo, R. L. Haupt, and T. W. Miller, Introduction to Adaptive Arrays, SciTech Publishing, 2nd edition, 2011.

[2] V. S. S. S. C. Vedula, S. R. C. Paladuga, and M. R. Prithvi, "Synthesis of circular array antenna for sidelobe level and aperture size control using flower pollination algorithm," International Journal of Antennas and Propagation, vol. 2015, Article ID 819712, 2015.

[3] Y. Gong, L. Wang, R. Yao, and Z. Zhang, "A robust method to suppress jamming for GNSS array antenna based on reconstruction of sample covariance matrix," International Journal of Antennas and Propagation, vol. 2017, pp. 1-12, 2017.

[4] D. R. Morgan and A. Aridgides, "Adaptive Sidelobe Cancellation of Wide-Band Multipath Interference," IEEE Transactions on Antennas and Propagation, vol. 33, no. 8, pp. 908-917, 1985.

[5] Q. Guo and L. Qi, "Cascaded multitype interferences suppression method using sparse representation and array processing for GNSS receiver," International Journal of Antennas and Propagation, vol. 2017, pp. 1-17, 2017. 
[6] S. Todnatee and C. Phongcharoenpanich, "Iterative GA optimization scheme for synthesis of radiation pattern of linear array antenna," International Journal of Antennas and Propagation, vol. 2016, Article ID 7087298, 2016.

[7] Y. Han and J. Wang, "Adaptive beam forming based on compressed sensing with smoothed," vol. 2015, 2015.

[8] I. El-Azhary, M. S. Afifi, and P. S. Excell, "Simple algorithm for sidelobe cancellation in a partially adaptive linear array," IEEE Transactions on Antennas and Propagation, vol. 36, no. 10, pp. 1484-1486, 1988.

[9] A. Trastoy, F. Ares, and E. Moreno, "Phase-only control of antenna sum and shaped patterns through null perturbation," IEEE Antennas and Propagation Magazine, vol. 43, no. 6, pp. 45$54,2001$.

[10] A. Trastoy and F. Ares, "Phase-only synthesis of continuous linear aperture distribution patterns with asymmetric side lobes," Electronics Letters, vol. 34, no. 20, pp. 1916-1917, 1998.

[11] M. M. Khodier and C. G. Christodoulou, "Linear array geometry synthesis with minimum sidelobe level and null control using particle swarm optimization," IEEE Transactions on Antennas and Propagation, vol. 53, no. 8, pp. 2674-2679, 2005.

[12] M. A. Mangoud and H. M. Elragal, "Antenna array pattern synthesis and wide null control using enhanced particle swarm optimization," Progress In Electromagnetics Research B, no. 17, pp. 1-14, 2009.

[13] M. Dawoud, A. Tennant, and A. Anderson, "Null steering in adaptive arrays using a genetic algorithm," in 24th European Microwave Conference, 1994, pp. 1108-1114, Cannes, France, October 1994.

[14] H. Steyskal, R. A. Shore, and R. L. Haupt, "Methods for null control and their effects on the radiation pattern," IEEE Transactions on Antennas and Propagation, vol. AP-34, no. 3, pp. 404409, 1986.

[15] S. K. Smith, J. C. Brégains, K. L. Melde, and F. Ares, "A comparison of optimization techniques for power patterns with low sidelobes generated by linear arrays with efficient excitation distributions," Microwave and Optical Technology Letters, vol. 45, no. 1, pp. 57-60, 2005.

[16] S. Kwak, J. Chun, D. Park, Y. K. Ko, and B. L. Cho, "Asymmetric sum and difference beam pattern synthesis with a common weight vector," IEEE Antennas and Wireless Propagation Letters, vol. 15, pp. 1622-1625, 2016.

[17] A. F. Morabito and P. Rocca, "Optimal synthesis of sum and difference patterns with arbitrary sidelobes subject to common excitations constraints," IEEE Antennas and Wireless Propagation Letters, vol. 9, pp. 623-626, 2010.

[18] J. R. Mohammed and K. H. Sayidmarie, "Null steering method by controlling two elements," IET Microwaves, Antennas \& Propagation, vol. 8, no. 15, pp. 1348-1355, 2014.

[19] C. A. Balanis, Antenna theory: Analysis and Design, John Wiley Sons, Hoboken, New Jersey, 3rd edition, 2005. 


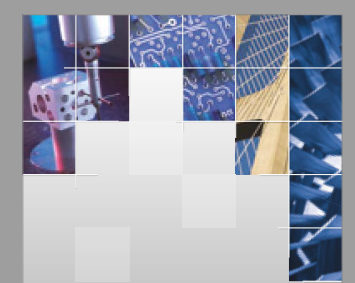

\section{Enfincering}
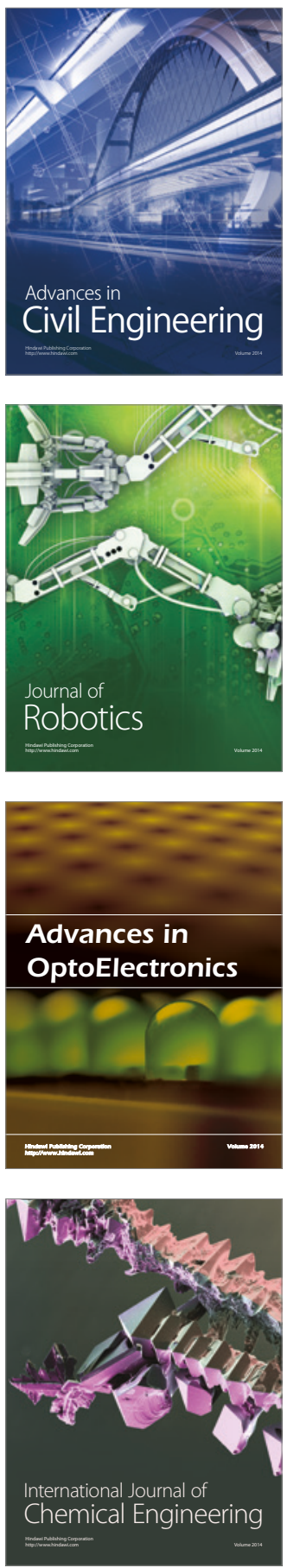

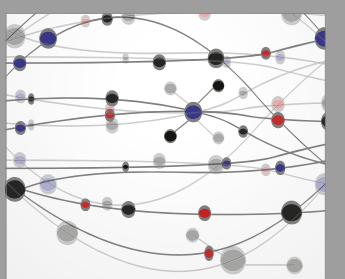

The Scientific World Journal

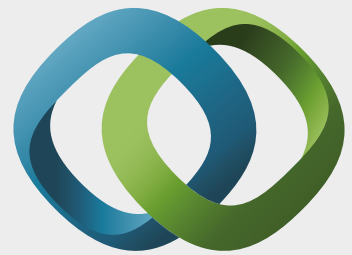

\section{Hindawi}

Submit your manuscripts at

https://www.hindawi.com
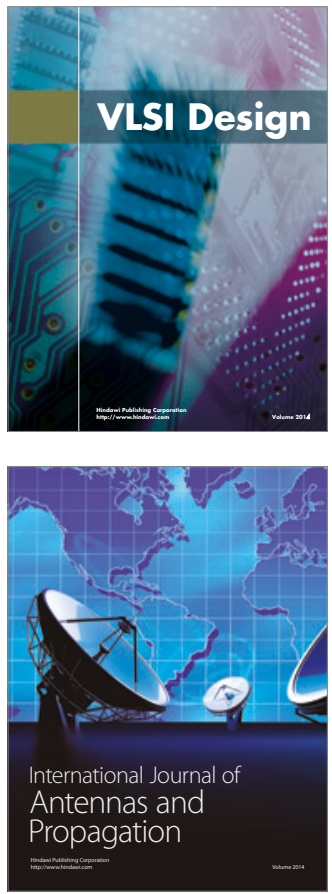

\section{Rotating}

Machinery
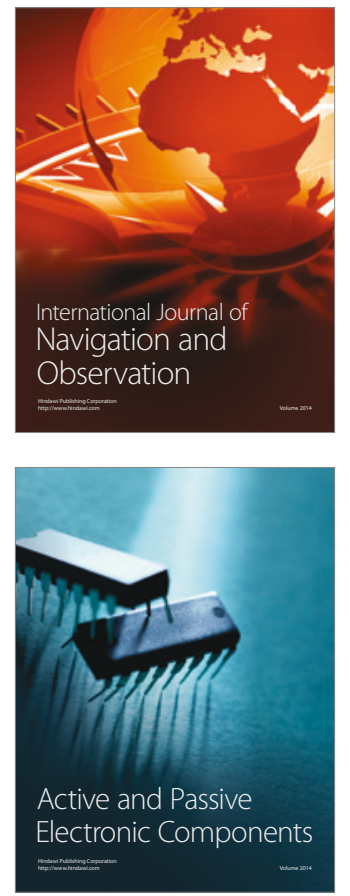
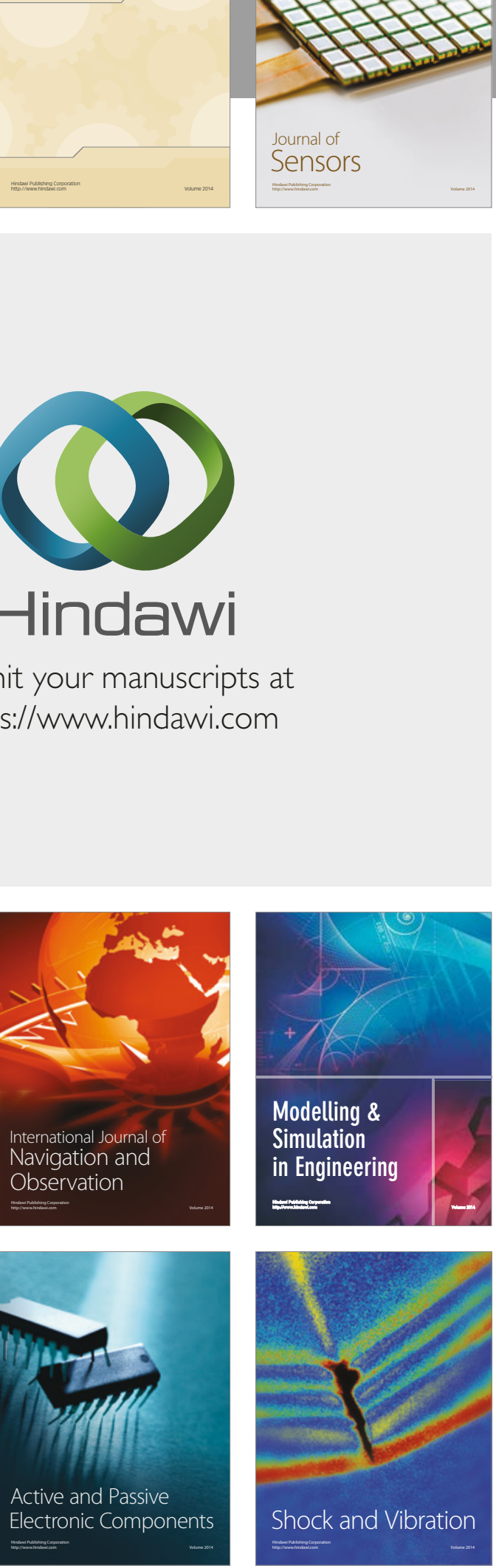
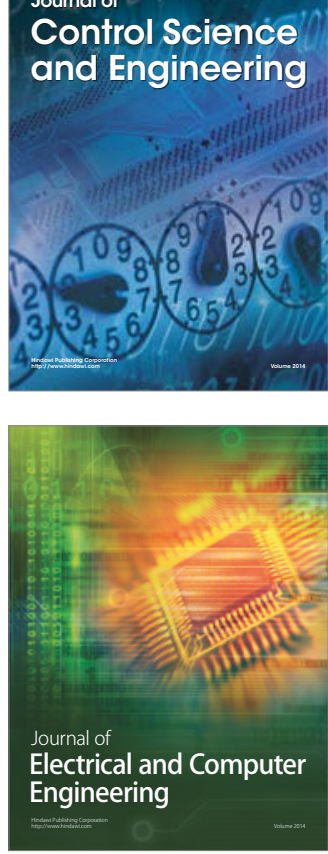

Distributed

Journal of

Control Science

and Engineering
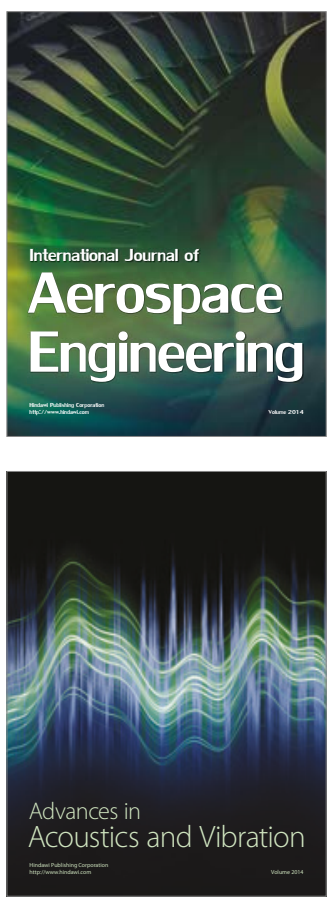

Sensor Networks 